\title{
Automated Pavement Distress Detection, Classification and Measurement: A Review
}

\author{
Brahim Benmhahe, Jihane Alami Chentoufi \\ Dept. of Informatics \\ Faculty of Sciences, Ibno Tofail University \\ Kenitra, Morocco
}

\begin{abstract}
Road surface distress is an unavoidable situation due to age, vehicles overloading, temperature changes, etc. In the beginning, pavement maintenance actions took only place after having too much pavement damage, which leads to costly corrective actions. Therefore, scheduled road surface inspections can extend service life while guaranteeing users security and comfort. Traditional manual and visual inspections don't meet the nowadays criteria, in addition to a relatively high time volume consumption. Smart City pavement management preventive approach requires accurate and scalable data to deduce significant indicators and plan efficient maintenance programs. However, the quality of data depends on sensors used and conditions during scanning. Many studies focused on different sensors, Machine Learning algorithms and Deep Neural Networks tried to find a sustainable solution. Besides all these studies, pavement distress measurement stills a challenge in Smarts Cities because distress detection is not enough to decide on maintenance actions required. Damages localization, dimensions and future development should be highly detected on real-time. This paper summarizes the state-of-the-art methods and technologies used in recent years in pavement distress detection, classification and measurement. The aim is to evaluate current methods and highlight their limitations, to lay out the blueprint for future researches. PMS (Pavement Management System) in Smarts Cities requires an automated pavement distress monitoring and maintenance with high accuracy for large road networks.
\end{abstract}

Keywords-Automated pavement distress detection; smarts cities; pavement management system; machine learning; deep neural networks

\section{INTRODUCTION}

Damages detection and measurement in bridges, buildings and roads are not a recent focus of researches. Multiple studies [22] were conducted during the last decades to determine adaptable equipment and solutions. However, [23] keeping pavement surfaces in a good condition via a low-cost solution is still a big challenge.

There is no doubt that concepts of the future [24] (Smarts Cities, Smarts Roads, Smart Traffic, Intelligent Transportation Systems, etc.) are looking for automation, precision, energyefficient and security while providing humans with the best services.

Nowadays, and besides the construction of multiple road networks, several cities are suffering congestion and overloading. For example [25], the number of private cars in China was 320 million in 2018; fifty-eight cities hold more than one million cars and seven cities support more than three millions.

Due to multiple causes, natural or human reactions, high qualities of roads surfaces are degraded and such situation cannot be avoided. When the severity of degradation becomes higher, it engenders multiple risks for road users and maintenance becomes costly and time-consuming. The run to failure concept is not the right solution and preventives actions can be conducted late or before it is necessary. The more accurate and efficient methods are those based on real-time monitoring [26], evaluating current status and estimating future development to decide on appropriate maintenance in every single timeslot.

Manual and visual inspection methods present an old concept. It takes more time, engages high manpower and presents multiple risks for inspectors and road users. For huge road networks, it is not practical at all to deploy inspectors along with the city to get a big amount of data, which is dynamic and space distributed.

PMS (Pavement Management System) is a concept that was adopted firstly in the State of California (USA) in 1979. The aim is to design a system taking all parameters that contribute to the pavement status into consideration and adapt, to the context, all methods and technologies to keep road surfaces in a good health.

Multiple types of sensors were used to collect data like vision, vibration, sound propagation, pressure, friction, etc. However, vision-based methods (images or videos) are more adaptable [21] because they overcome weather limitations and scan the whole area, which is not always possible for the remaining methods.

This paper is organized as follow. Section II describes PMS and its contribution to Smarts Cities development. Section III presents related works to pavement distress detection and measurement. In Section IV, experiences and results of current methods are depicted. In Section V, current limitations and futures research are highlighted. Before concluding the paper on Section VII, a discussion is presented in Section VI.

\section{PAVEMENT MANAGEMENT SySTEM}

\section{A. Concept}

As long as cities become larges, an automated system to manage pavement condition becomes a reel need. In 1985, the American Association of State Highway and Transportation 
Officials (AASHTO) was the first agency that defines the PM (Pavement Management), as: "The effective and efficient directing of the various activities involved in providing and sustaining pavements in a condition acceptable to the traveling public at the least life cycle cost”. PM or PMS doesn't make decisions [28]; it just helps agencies to choose the best solution through the evaluation of alternative decisions.

\section{B. Terms}

The AASHTO [29] defined some terms relative to the Pavement Management as summarized in Table I.

TABLE I. PAVEMENT MANAGEMENT TERMS \& DEFINITIONS BY AASHTO GUIDELINES

\begin{tabular}{|c|c|}
\hline Term & Definition \\
\hline $\begin{array}{l}\text { Pavement } \\
\text { Management (PM) }\end{array}$ & $\begin{array}{l}\text { A program for improving the quality and performance } \\
\text { of pavements and minimizing costs through good } \\
\text { management practices. }\end{array}$ \\
\hline $\begin{array}{l}\text { Pavement } \\
\text { Management } \\
\text { System (PMS) }\end{array}$ & $\begin{array}{l}\text { A set of defined procedures for collecting, analyzing, } \\
\text { maintaining, and reporting pavement data, to assist the } \\
\text { decision makers in finding optimum strategies for } \\
\text { maintaining pavements in serviceable condition over a } \\
\text { given period of time for the least cost. }\end{array}$ \\
\hline $\begin{array}{l}\text { Pavement } \\
\text { Management } \\
\text { Information } \\
\text { System (PMIS) }\end{array}$ & $\begin{array}{l}\text { An established and documented procedure for } \\
\text { collecting, storing, processing, and retrieving the } \\
\text { information required in a PMS. It represents a } \\
\text { foundation for PMS since all pavement decisions must } \\
\text { be based on a common, integrated source of } \\
\text { information derived from reliable and good quality } \\
\text { data. }\end{array}$ \\
\hline $\begin{array}{l}\text { Pavement } \\
\text { Condition }\end{array}$ & The present status or performance of a pavement. \\
\hline $\begin{array}{l}\text { Pavement } \\
\text { Maintenance } \\
\text { Techniques } \\
\text { Methods }\end{array}$ & $\begin{array}{l}\text { Used to accomplish strategy or correct deficiency in } \\
\text { pavement segment. }\end{array}$ \\
\hline $\begin{array}{l}\text { Pavement } \\
\text { Management } \\
\text { Strategy }\end{array}$ & $\begin{array}{l}\text { A carefully arranged, systematic program of action } \\
\text { applied to any area of pavement activity. }\end{array}$ \\
\hline $\begin{array}{l}\text { Pavement } \\
\text { Performance }\end{array}$ & $\begin{array}{l}\text { The assessment of how well the pavement serves the } \\
\text { user over time. The engineer often associates pavement } \\
\text { condition with an arbitrary, but quantifiable, value } \\
\text { relating to pavement roughness, pavement distress, or } \\
\text { pavement strength. Performance is the measured } \\
\text { change of condition and/or serviceability over } \\
\text { increments of time. }\end{array}$ \\
\hline $\begin{array}{l}\text { Pavement } \\
\text { Optimization }\end{array}$ & $\begin{array}{l}\text { A procedure for obtaining the greatest life-cycle } \\
\text { benefits for the lowest cost. Within the practice of } \\
\text { Pavement Management, optimization might best be } \\
\text { described as a process of obtaining the highest state of } \\
\text { pavement performance over the pavement's life cycle } \\
\text { with the least social and economic impact. }\end{array}$ \\
\hline
\end{tabular}

\section{Pavement Management System Implementation}

To implement PMS in a special context, several models were proposed in the literature. Illinois Center for Transportation [27] proposed a model as follow:

- Step 1: Define the Roadway Network and Collect Inventory Data.
- Step 2: Collect Condition Data.

- Step 3: Predict Condition.

- Step 4: Select Treatments.

- Step 5: Report Results.

- Step 6: Select Pavement Management Tool.

- Step 7: Keep the Process Current.

\section{Pavement Damages Classification}

1) Pavement damages types: It should be determined to apply the appropriate maintenance. Distress shapes can be caused by factors mentioned in Section I. Pavement distress can be categorized as mentioned in Table II.

TABLE II. PAVEMENT DistRESS CATEgorization

\begin{tabular}{|c|c|}
\hline \multirow{7}{*}{ Cracking } & Fatigue Cracking (Alligator Cracking) \\
\hline & Block Cracking \\
\hline & Edge Cracking \\
\hline & Longitudinal Cracking \\
\hline & Transverse Cracking \\
\hline & Reflection Cracking \\
\hline & Slippage Cracking \\
\hline \multirow{5}{*}{ Distortion } & Rutting \\
\hline & Shoving \\
\hline & Depressions \\
\hline & Upheaval \\
\hline & Patch Failures \\
\hline \multirow{2}{*}{ Disintegration } & Raveling \\
\hline & Potholes \\
\hline \multirow{2}{*}{ Skidding Hazards } & Polished Aggregate \\
\hline & Bleeding \\
\hline \multirow{2}{*}{$\begin{array}{l}\text { Surface Treatment } \\
\text { Distress }\end{array}$} & Surface Treatment Distress \\
\hline & Streaking \\
\hline
\end{tabular}

2) Pavement damages severity: Type of damage is not enough to define maintenance actions. Damage severity should also be measured.

Multiple documents defined models to categorize severity levels (low, medium, high, etc.). Categorization could be different from document to other.

R. Roberts studied and analyzed six documents of pavement distress classification methods [11]: American Society for Testing \& Materials (ASTM) 2018, Miller \& Bellinger 2014, British Columbia Ministry of Transportation \& Infrastructure Construction Maintenance Branch 2016, Bertrand et al. 1998, Direzione Generale Infrastructure eMobilità Milano 2005 and VicRoads 2009. Analyzing the aforementioned documents reveals that distress evaluated as 
medium by a manual could be classified as lower or higher by other. Furthermore, some manuals defined the severity based on the distress dimensions and the ratio of the distressed area to the total surface square (ASTM, 2018). However, others use only the ratio parameter.

\section{E. Pavement Surface Evaluation}

The evaluation of pavement surface health is a combination of type and severity level of damages. Literature review analysis [17] indicates that the most indices used for pavement surface evaluation are:

- Pavement Condition Index (PCI);

- International Roughness Index (IRI);

- Ruth Depth (RD);

- Pavement Serviceability Index (PSI).

\section{F. Pavement Maintenance}

A.M. Johnson [7] has defined three types of pavement maintenance:

1) Preventive maintenance: Performed to improve or extend the functional life of a pavement. It is a strategy of surface treatments and operations intended to retard progressive failures and reduce the need for routine maintenance and service activities.

2) Corrective maintenance: Performed after a deficiency occurs in the pavement, such as loss of friction, moderate to severe rutting, or extensive cracking. It may also be referred to as "reactive" maintenance.

3) Emergency maintenance: Performed during an emergency situation, such as a blowout or severe pothole that needs repair immediately. This also describes temporary treatments designed to hold the surface together until more permanent repairs can be performed.

Predictive Maintenance is another concept adopted for pavement distress using Machine Learning, ANN (Artificial Neural Networks), or other algorithms to predict the future health of pavement. Despite the limitation of current algorithms, prediction models present a cost-effective concept that keeps surface roads healthy almost every time.

\section{RELATED WORK}

\section{A. Materiel and Equipment in use}

1) Legacy methods: Visual and manual inspection methods of pavement surfaces were conducted by road inspectors using traditional tools. Almost data collected is suffering from subjectivity, not containing all parameters needed and presents a low quality. In this case, data collected is costly and time-consuming. Furthermore, infantile damages can't be detected and still presents for a considerable amount of time until maintenance actions become heavy [1]. Otherwise, during a survey, the safety of inspectors and road users is highly engaged.

2) New technologies: Several sensors and equipment were used to evaluate pavement surface conditions by collecting different parameters [21]: image/video, acceleration, vibration, sound, pressure, friction, skid resistance, etc. During the last years, Condition Based Monitoring (CBM) was applied to pavement surface evaluation. Y. Hou summarizes intrusive sensors development and installation, as embedded equipment, in the pavement structure [26]. In this case, sensors are used to monitor the dynamic mechanical response of the pavement to vehicle load and weather influence. Data collected are transferred by the Internet of Things (IoT) technologies. Table III summarizes and categorizes sensors used.

TABLE III. SENSORS USED ON PAVEMENT Distress Data ColLection

\begin{tabular}{|c|c|c|}
\hline $\begin{array}{l}\text { Parameters } \\
\text { collected }\end{array}$ & Equipment used & Location of installation \\
\hline \multirow{4}{*}{ Image/ Video } & $\begin{array}{l}\text { Camera: CCD, CMOS, } \\
\text { Infrared }\end{array}$ & Fixed around pavement. \\
\hline & Smartphone & \multirow{2}{*}{$\begin{array}{l}\text { Mounted on vehicle; Moved } \\
\text { by operator. }\end{array}$} \\
\hline & Line Scan & \\
\hline & UAV & Flying \\
\hline Acceleration & $\begin{array}{l}\text { Smartphone; } \\
\text { Accelerometer }\end{array}$ & $\begin{array}{l}\text { Mounted on vehicle : wheel } \\
\text { axis, in-car }\end{array}$ \\
\hline Vibration & Vibration sensor & $\begin{array}{l}\text { Mounted on vehicle: wheel } \\
\text { axis, in-car }\end{array}$ \\
\hline 3D Data & $\begin{array}{l}\text { Laser Profiler; } \\
\text { Line Projection; } \\
\text { Stereovision; } \\
\text { Kinect Device; Ground } \\
\text { Penetrating Radar; } \\
\text { Structured Light; } \\
\text { Photometric Stereo }\end{array}$ & $\begin{array}{l}\text { Mounted on vehicle; } \\
\text { Moved by operator. }\end{array}$ \\
\hline Sound & Microphone & $\begin{array}{l}\text { Mounted on vehicle: on tire, } \\
\text { beneath the car; } \\
\text { Fixed around pavement. }\end{array}$ \\
\hline $\begin{array}{l}\text { Friction } \\
\text { and } \\
\text { Skid } \\
\text { resistance }\end{array}$ & $\begin{array}{l}\text { Tire (Traction); Wheel } \\
\text { (Angled) }\end{array}$ & Mounted on vehicle \\
\hline Pressure & Pressure sensor & \multirow{7}{*}{$\begin{array}{l}\text { Embedded in pavement } \\
\text { structure }\end{array}$} \\
\hline Temperature & Temperature sensor & \\
\hline Humidity & Humidity sensor & \\
\hline Stress & Stress sensor & \\
\hline strain & Strain sensor & \\
\hline Displacement & Displacement sensor & \\
\hline Light & Light sensor & \\
\hline
\end{tabular}

\section{B. Datasets}

Data collected by sensors create datasets to train, evaluate and test algorithms. Multiple datasets are now available and open access. The quality of the dataset is determined by its volume, robustness, and exhaustiveness by containing data: 
- Variable and complex;

- Collected under different weather conditions ;

- Collected with different lighting and noise levels;

- Blurry and degraded with high sample densest.

Datasets collected by acceleration and vibration sensors installed on cars do not handle all pavement surfaces. In this case, sensors collect only damages crossed by wheels' cars.

Datasets annotation adds information about distress types. Annotations can be done manually or by using algorithms (ex. Python). Some Datasets are annotated at the pixel level, which gives a clear idea about pavement condition and enhances the precision of algorithms.

We can make the dataset more exhaustive by applying the "Data Augmentation" method to the images collected. J. Liu [15] used image rotation $\left(90^{\circ}, 180^{\circ}, 270^{\circ}\right)$ and flipping $\left(180^{\circ}\right.$ horizontally and vertically) to get 7104 images from 1184 initially captured.

1) Vision datasets: Nowadays, it was proved that images/videos collection is more adaptable for pavement surface conditions [21]. Table IV summarizes datasets images of pavement. Besides the datasets mentioned, several researchers have collected specific datasets to test algorithms (ex. Crackdataset [2]). Those datasets were reserved for studies. Images extracted from videos give more information about damages. GAPs, GaMM and EdmCrack600 are a datasets containing video imaging.

TABLE IV. PAVEMENT CONDITION DATASETS

\begin{tabular}{|l|l|l|l|l|}
\hline Dataset Name & $\begin{array}{l}\mathbf{N}^{\circ} \text { Of } \\
\text { Images } \downarrow\end{array}$ & Resolution & Device & Privacy \\
\hline $\begin{array}{l}\text { Street View } \\
\text { Images }\end{array}$ & 9712 & --- & --- & Private \\
\hline $\begin{array}{l}\text { NEU Inlaid } \\
\text { Crack }\end{array}$ & 9396 & 256 X 256 & $\begin{array}{l}\text { CCD camera } \\
\text { \& light }\end{array}$ & Private \\
\hline Japan Road & 9053 & $600 \times 600$ & LG Nexus 5X & Public \\
\hline PID & 7237 & $640 \times 640$ & $\begin{array}{l}\text { Google API } \\
\text { Street-View }\end{array}$ & Public \\
\hline GPR Images & 6832 & 256 X 256 & LTD-2000 & Private \\
\hline $\begin{array}{l}\text { 3D Asphalt } \\
\text { Surface } \\
\text { Images }\end{array}$ & 2000 & 4096 X 2048 & PaveVision3D & Private \\
\hline GAPs & 1969 & $1920 \times 1080$ & $\begin{array}{l}\text { Professional } \\
\text { Camera }\end{array}$ & Public \\
\hline FHWA/LTPP & 1056 & $3072 \times 2048$ & --- & Public \\
\hline Local & 800 & $2000 \times 4000$ & --- & Private \\
\hline EdmCrack600 & 600 & $1920 \times 1080$ & GoPro 7 & Public \\
\hline Crack500 & 500 & $2000 \times 1500$ & LG-H345 & Public \\
\hline Cracktree200 & 206 & $800 \times 600$ & --- & Public \\
\hline CFD & 118 & $480 \times 320$ & iPhone 5 & Public \\
\hline CrackIT & 84 & 1536 X 2048 & Optical & Public \\
\hline GaMM & 42 & $1920 \times 480$ & $\begin{array}{l}\text { Professional } \\
\text { camera }\end{array}$ & Public \\
\hline Aigle-RN & 38 & $991 \times 462$ & $\begin{array}{l}\text { Professional } \\
\text { camera }\end{array}$ & Public \\
\hline
\end{tabular}

2) $U A V$ datasets: Some studies [10, 20, 21] were used UAVs (Unmanned Aerial Vehicles) to inspect pavement condition. Aerospace imagery was applied in several domains (agricultural, mapping, forestry, surveillance, etc.) and proved valuable.

UAVs present the advantages of mobility along the third dimension, flexibility to change trajectory in flight, discretion, avoiding human risks and remote controlling. GSD (Ground Sampling Distance) is a parameter that defines the spatial resolution of images. Practically, it is the distance between adjacent pixel centers measured on the ground. Equation (1) shows the formula of GSD:

$G S D=\frac{D \times P x}{f}$

D: distance between UAV and ground

Px: pixel size

f: camera focal length

A low value of GSD means a high quality of image. The quality of UAV images increased with high camera resolution or low flight altitude.

\section{Extraction and Analysis}

Several algorithms (ANN, Deep Neural Networks and Machine Learning) were developed and tested to extract meaningful information from datasets. In this section, we will review articles published since January 2020 to evaluate the last researches in this domain.

1) Camera/smartphone images extraction: Q. Mei [1] presents a new Deep Learning algorithm for pavement cracks segmentation "DenseCrack". The algorithm was trained and tested on two datasets Crack Forest Dataset (CFD) and Imagenet.

W. Song [2] proposed CrackSeg, which is a Deep Convolutional Neural Network (CNN) for pavement cracks detection. CrackSeg was trained, evaluated and tested on CrackDataset which contains 8188 images and was dedicated to the study. CrackSeg was also tested on CFD and AigleRN.

R. Roberts [3] makes the conception and the realization of a Low-Cost Data Acquisition and Analysis System for PMS in 3 phases:

- Phase 1: Pavement images collection by using two smartphones and one camera. The distance from the pavement surface was $1500 \mathrm{~m}$.

- Phase 2: Conversion of images collected to 3D model by using SFM (Structure From Motion) technique.

- Phase 3: Analyzing images and Points Cloud by Cloud Compare.

- Phase 4: Points Cloud segmentation by using RANSAC and Fit algorithms.

Z. Tong [4] makes a combination of an FCN (Fully Convolutional Network) and a GCRF (Gaussian Conditional Random Field) to develop a new technique of pavement 
distress detection. This method enhances the precision of the detection by extracting more information about distress type, localization and dimensions. The algorithm contains a framework of incertitude calculation and a probability-based role to reject wrong segmentation. A dedicated dataset for algorithm training, validation and testing was collected by Datong SH5047XJCA2D5 (vehicle equipped with cameras). The dataset contains 8820 images collected under different resolutions and formats by a vertical camera to the pavement surface.

Y. Du [5] used YOLO Network v3 to create a method of the detection and the classification of pavement damages. A dataset of pavement images was prepared using a camera installed on a vehicle. 45788 images were captured along 200 $\mathrm{KM}$ of pavement under different weather and lighting conditions.

Z. Fan [6] suggested a group of CNNs based on Probability Fusion for automatic detection and measurement of pavement cracks. This method consists of three steps:

- Step 1: a group of CNNs was used to identify the structure of small cracks on raw images.

- Step 2: the average of all networks' outputs gives the probability of cracks on each pixel.

- Step 3: Skeleton algorithm measures the prediction of cracks' characteristics.

The proposed method was validated and tested on two datasets CFD and AigleRN. It can measure the width and the depth of different cracks (complex, thin, and intersecting cracks) based on a Crack Maps Prediction.

X. Xiang [9] proposed a new method for cracks detection based on an end-to-end trainable Deep CNN. The network was trained on Crack500 and evaluated on three datasets Crack500, Cracktree200 and CFD.

Q. Mei [12] proposed a cost-effective solution for pavement cracks inspection using a Grade Sport GoPro camera mounted on a vehicle. Two configurations of camera installation were studied (rear and front). Rear configuration was more advantageous for the following reasons:

- Light reflection from the windshield inside the car reduces image quality for front configuration.

- Front camera is relatively far from the pavement surface.

- FOV (Field Of View) of the front camera is reduced by the car's hood.

- Using backup cameras of cars in future researches to optimize equipment needed.

A new crack detection method ConnCrack was developed, which combines a Conditional Wasserstein Generative Adversarial Network and a Connectivity Maps. ConnCrack was pre-trained on two public datasets ImageNet and CFD. Then, it was trained and tested on the EdmCrack600 dataset collected for the study.
J. Liu [15] makes the conception of a method for pavement crack detection and segmentation based on a CNN in two steps:

- Step 1: automatic cracks detection using an algorithm based on YOLO V3 (modified).

- Step 2: cracks segmentation using an algorithm based on U-Net (modified).

Cracks detected during the first step will be segmented on the second step to enhance the method precision. A dataset of pavement crack images was collected for the study under different weather conditions and noise (stains, pavement markings, sealed pavement, sand, leaves, branches, shadow, etc.). 1066 images were collected by a smartphone (dual cameras 13 Megapixels) at a fixed distance from the pavement (1.5 m). CFD dataset images (118) were added to the study dataset to get 1184 images. Then, data augmentation was applied to get 7104 images. Both methods (Step 1 \& Step 2) were trained, evaluated and tested on the global dataset.

E. Ibragimov [18] proposes a method for automatic pavement distress detection based on Faster Region Convolutional Neural Network (Faster R-CNN). The study focused on four types of cracks: Longitudinal, Transverse, Alligator and Partial Patching. The validation of the method was conducted on a dedicated dataset collected for the study. Dataset (3 200 images) was collected by a high-resolution camera mounted on a vehicle moving at a speed of $30 \mathrm{~km} / \mathrm{h}$ along $10 \mathrm{~m} * 3.7 \mathrm{~m}$ of pavement.

Y. Wang [19] creates a framework RENet "Rectangular convolution pyramid and edge enhancement Network" for pavement cracks detection. RENet is based on the modified ResNet-18 network, which is pre-trained on the ImageNet. The framework was tested on two datasets CRACK500 and NEU inlaid Crack and compared to 7 other methods (SMD, BC, MIL, Yin, NLDF, FCN, FPHB and SRM) on the same datasets.

2) Vision-based vs Vibration-based: J. Lekshmipathy [8] makes a conception and a comparison of two automatic methods of pavement distress detection, one based on vision and the other on vibration. All experiences for both methods were conducted on the same road segment $(6.2 \mathrm{~km})$, and the results were validated by a manual survey. For the vibration method, accelerometers and gyroscope of a smartphone were used to collect data, and an Artificial Neural Network Technique was used to detect and classify pavement distress. For the vision method, data was collected by a camera (Sony Handycam, 8.9 Megapixels) mounted on the rear end of a car; images were captured at a speed between 10 and $15 \mathrm{~km} / \mathrm{hr}$. Pavement distress detection and extension were conducted using a MATLAB code.

3) UAV images extraction: M. Zeybek [10, 20] used the mini UAV DJI Phantom 4 RTK (P4RTK) to inspect the condition of a road segment (100Meters) contains small cracks, potholes and ruts. The proposed method constructed on three phases: 
- Phase 1: collection of images by a flying UAV.

- Phase 2: generation of Point Cloud (3D) and Orthomosaic with the SFM Pix4DMapper.

- Phase 3: damage measure (diameter, perimeter, width and depth) conducted on 2D and 3D data by Global Mapper Software.

R. Roberts [21] creates 3D models from UAV images by using SFM technique. The UAV used is the DJI Mavic 2 Pro (Commercial) with a 907g weight and 20Megapixels camera resolution. During data collection, 554 images were collected with a distance of $8 \mathrm{~m}$ from the ground and a GSD of $0.97 \mathrm{~mm} /$ pixel. RANSAC (RANdom SAmple Consensus) and 2.5D Quadric Fit algorithms were used to segment images and evaluate the degradation levels.

4) GPR images extraction: J. Gao [13] makes the conception of Faster R-ConvNet (Region- Convolutional Neural Network), which is a Deep Learning method for pavement distress detection. Faster R-ConvNet was trained, validated and tested using 3785 GPR (Ground Penetrating Radar) images. Images were collected using LTD-2000 GPR (made in China) and containing different damages: Reflection Crack, Water-Damage-Pit and Uneven Settlement. During images collection, different frequencies were used to guarantee dataset integrality.

M. E. Torbaghan [14] proposed a method for automatic detection of cracks on road surfaces using GPR and established the limits of minimal dimensions detectable. The Singular Value Decomposition (SVD) algorithm reduces clutters on images. Then, a filter is applied to eliminate random noises. GPR images collection by Ground View 3 GPR (Utsi Electronics, $2 \mathrm{GHz}$ antenna) were conducted on seven pavement slabs samples. In total, $3.5 \mathrm{~m}$ length of pavement contains 14 different cracks. Each slab is $500 \mathrm{~mm} \times 500 \mathrm{~mm} \times$ $110 \mathrm{~mm}$ dimension. To test the ability of the system to detect pavement damages under environmental and physical conditions change, the following parameters were simulated:

- Cracks' surface was covered with paper to simulate the coverage of cracks by leaves’ plants.

- Cracks were filled with thin layers of Bitumen to test the ability of the system to detect voids and cracks that do not penetrate to the surface.

- Cracks were filled with fine grains of sand to simulate debris and dust.

5) Transfer learning: S. Ranjbar [16] adopted the Transfer Learning technique to $\mathrm{ML}$ algorithms in order to solve the problem of a big data need for training. He trained, on a dataset, the Pre-Trained Models: AlexNet, GoogleNet, SqueezNet, ResNet-18, ResNet-50, ResNet-101, DenseNet201 and Inception-v3. The dataset contains 1500 images of pavement Linear Cracking, Surface Cracking and NonCracking.

\section{EXPERIENCES AND RESULTS}

In order to make a significant comparison between algorithms, some indicators were defined and measured. The most indicators used are Precision, Recall and F1 Score.

\section{A. Indicators Definitions}

- Precision: the percentage of real cracks detected (TP) by a method from the total of real cracks existing (TP+FP) (2):

$\operatorname{Pr}=\frac{T P}{T P+F P}$

- Recall: the percentage of reality (TP) on all cracks detection by a method $(\mathrm{TP}+\mathrm{FN})(3)$ :

$R e=\frac{T P}{T P+F N}$

- F1 Score: is the harmonic average value of precision and recall (4):

$F 1$ Score $=\frac{2}{1 / P r+1 / R e}$

During algorithm tests on a dataset, damages detection is compared to the real damages confirmed by dataset labels. However, it seems that some real damages are not detected by the algorithm, and some damages detection by the algorithm is not real. Damages accuracy verification can be done at the region or the pixel level. Finally, four situations can be defined:

S1: Picture/Pixel damaged according to the dataset and the algorithm; is counted as TP (True Positive).

S2: Picture/Pixel damaged according to the dataset but not detected by the algorithm; is counted as FP (False Positive).

S3: Picture/Pixel not damaged according to the dataset but supposed damaged by the algorithm; is counted as FN (False Negative).

S4: Picture/Pixel not damaged according to the dataset and the algorithm; is counted as TN (True Negative).

For Picture or Pixel level reasoning, the totals of counted TP, FP, FN and TN are calculated. Precision (Pr), Recall (Re) and F1 Score are calculated using (2), (3) and (4) respectively.

\section{B. Cracks Detection}

Several algorithms for cracks detection were proposed by the literature. To compare the performance of these algorithms, values of indicators (Precision, Recall, F1 Score) were collected and reviewed from publications. Table V summarizes indicators of the most popular cracks detection algorithms on the four datasets: CFD, CrackDataset, Aigle RN and EdmCrack600.

On the CFD dataset, the two steps concept based on the CNN proposed by J. Liu [15] get the best values of $\mathrm{Pr}$ (97.24\%) and F1 score (95.75\%). On the same dataset, the best value of Re $(95.70 \%)$ were achieved by U-Net method based on Pixel-level crack detection [15]. 
On CrackDataset, CrackSeg achieved the best results on the three indicators: $\mathrm{Pr}=98.00 \%, \quad \mathrm{Re}=97.85 \%$ and $\mathrm{F} 1$ score $=97.92 \%$.

On Aigle-RN dataset, Z. Fan [6] achieves the best values of the three indicators: $\operatorname{Pr}=93.02 \%, \mathrm{Rec}=91.66 \%$ and $\mathrm{F} 1$ score $=$ $92.38 \%$.

On EdmCrack600, best values were achieved by ConnCrack [12]: $\operatorname{Pr}=80.88 \%, \quad \operatorname{Re}=91.66 \%$ and $\mathrm{F} 1$ score $=92.38 \%$.

\section{Non-Cracks Distress Detection and Analysis}

Other methods were not limited to cracks studies, but they open the scope to analyze other damages: Pothole, Patching, Ruts, Manhole, etc.

R. Roberts [3] creates a low-cost imagery model for detecting and analyzing pavement distress. Images were collected using one camera (Nikon D5200) and two
Smartphones (Huawei P20Pro and Samsung GalaxyS9). The accuracy of using mobile images to create 3D models was proved [3]. To evaluate smartphones' utility, Weibull parameters were measured (Shape and Scale) for the three distress surveys of the study, as illustrated in Table VI.

Y. Du [5] makes the conception of a method for pavement distress detection and classification based on YOLO Network v3. The method was tested on the dataset collected for the study, which contains three sets: 10000,20000 and 30000 images set. Best values were achieved on the 30000 images set. For Manhole distress (9 567/30 000 images), AP=92.7\% and F1 $=93.20 \%$ which represent the higher values. Minor values were relative to Potholes detection (1093/30 000 images) with $\mathrm{AP}=60.2 \%$ and $\mathrm{F} 1=67.03 \%$. On the same dataset, the algorithm was compared to Faster R-CNN and SSD using AP (Average Precision), F1 Score and operation time indicators (Table VII).

TABLE V. CRACKS DETECTION ALgORITHMS COMPARISON ON FOUR DATASETS

\begin{tabular}{|c|c|c|c|c|c|c|c|c|c|c|c|c|}
\hline \multirow{2}{*}{ Algorithm } & \multicolumn{3}{|c|}{ CFD (\%) } & \multicolumn{3}{|c|}{ CrackDataset [2] (\%) } & \multicolumn{3}{|c|}{ Aigle RN (\%) } & \multicolumn{3}{|c|}{ EdmCrack600 [12] (\%) } \\
\hline & Pr & Re & F1 & Pr & $\mathbf{R e}$ & F1 & Pr & $\mathbf{R e}$ & F1 & Pr & Re & F1 \\
\hline Dense Crack 201 & 92.02 & 91.13 & 91.58 & & & & & & & & & \\
\hline Canny & 43.77 & 73.07 & 45.70 & & & & 19.89 & 67.53 & 28.81 & 1.69 & 34.17 & 3.14 \\
\hline FFA & 78.56 & 68.43 & 73.15 & & & & 76.88 & 68.12 & 68.17 & & & \\
\hline CrackTree & 73.22 & 76.45 & 70.80 & & & & & & & & & \\
\hline CrackForest (SVM) & 82.28 & 89.44 & 85.71 & 86.28 & 85.46 & 85.86 & 90.28 & 86.58 & 88.39 & & & \\
\hline MFCD & 89.90 & 89.47 & 88.04 & & & & & & & & & \\
\hline CrackNet-V & 92.58 & 86.03 & 89.18 & & & & & & & & & \\
\hline U-Net & 92.54 & 89.51 & 89.90 & 96.99 & 97.09 & 97.04 & & & & 76.33 & 70.88 & 71.52 \\
\hline Z. Fan (2020) [6] & 95.52 & 95.21 & 95.33 & & & & 93.02 & 91.66 & 92.38 & & & \\
\hline Local Thresholding & 77.27 & 82.74 & 74.18 & & & & 53.29 & 93.45 & 66.70 & & & \\
\hline Structured Prediction & 92.27 & 94.89 & 93.12 & & & & 91.88 & 88.61 & 90.21 & & & \\
\hline ResNet152-FCN & 87.83 & 88.19 & 88.01 & & & & & & & 78.98 & 56.51 & 62.78 \\
\hline VGG19-FCN & 92.80 & 85.49 & 88.53 & & & & & & & 80.22 & 59.93 & 65.18 \\
\hline ConnCrack [12] & 96.79 & 87.75 & 91.96 & & & & & & & 80.88 & 76.64 & 76.98 \\
\hline Crack IT & 67.23 & 76.69 & 71.64 & & & & 76.85 & 74.32 & 76.56 & 12.33 & 7.14 & 4.75 \\
\hline J. Liu [15] (Two Steps) & 97.24 & 94.31 & 95.75 & & & & & & & & & \\
\hline Ai. Jiang (2018) & 90.70 & 84.60 & 87.00 & & & & & & & & & \\
\hline Jenkins et al. (2018) & 92.64 & 82.82 & 87.38 & & & & & & & & & \\
\hline Bang et al. (2019) & 93.57 & 84.90 & 89.03 & & & & & & & & & \\
\hline Nguyen et al. (2018) & 93.06 & 89.31 & 91.14 & & & & & & & & & \\
\hline Fan et al. (2018) & 91.19 & 94.81 & 92.44 & & & & & & & & & \\
\hline Cheng et al. (2018) & 92.12 & 95.70 & 93.88 & & & & & & & & & \\
\hline
\end{tabular}

TABLE VI. WEIBULL PARAMETERS IN THE Three Distresses SuRVEyed [3]

\begin{tabular}{|l|l|l|l|l|l|l|}
\hline \multirow{2}{*}{ DEVICE } & DISTRESS 1 & DISTRESS 2 & DISTRESS 3 \\
\cline { 2 - 7 } & Shape & Scale & Shape & Scale & Shape \\
\hline Huawei P20 Pro & 1.186156 & 0.002275 & 0.941246 & 0.001772 & 0.725207 \\
\hline Samsung Galaxy S9 & 0.981589 & 0.002794 & 1.005422 & 0.001528 & 1.183398 \\
\hline
\end{tabular}

TABLE VII. COMPARISON BETWEEN YOLOv3, FASTER R-CNN AND SSD ON THE COLLECTED DATASET [5]

\begin{tabular}{|l|l|l|l|}
\hline Distress Type & Higher AP & Higher F1 Score & Minor Operation Time \\
\hline Crack & Faster R-CNN $: 49.3 \%$ & Faster R-CNN $: 54.88 \%$ & \\
\hline Patch-Crack & Faster R-CNN $: 72.3 \%$ & Faster R-CNN $: 76.58 \%$ & \multirow{2}{*}{ YOLOv3 (Batch = 64) } \\
\hline Pothole & YOLOv3 (Batch $=64): 38.6 \%$ & YOLOv3 (Batch $=96): 40.88 \%$ & \\
\hline Patch-Pothole & YOLOv3 (Batch $=64): 63.3 \%$ & YOLOv3 (Batch $=64): 68.55 \%$ & \\
\hline Net & Faster R-CNN $: 53.5 \%$ & Faster R-CNN $: 56.90 \%$ & \\
\hline Patch-Net & Faster R-CNN $: 41.9 \%$ & Faster R-CNN $: 44.32 \%$ & \\
\hline Manhole & Faster R-CNN : 86.1\% & Faster R-CNN $: 90.45 \%$ & \\
\hline
\end{tabular}


Faster R-CNN makes good performances on detecting Cracks, Patch-Cracks, Nets, Patch-Nets and Manholes. However, YOLOv3 makes good performances on Pothole and Patch-Pothole detection and makes minor Operation time for all distress types detection (365/S).

E. Ibragimov [18] develops an algorithm for automatic pavement damage detection based on Faster R-CNN. The method was tested on a dataset collected for the study that contains three types of damage: Linear cracks, area cracks and patching. The method makes good performances on detecting area cracks and patching. On patching detection, Precision, Recall, and F1 Score were respectively 84.00\%, 88.09\% and 87.21\%. Difficulties were occurred on the detection of Linear Cracks (Longitudinal and Transverse): Pr=38.15\%, $\mathrm{Re}=41.23 \%$ and $\mathrm{F} 1=31.86 \%$.

\section{Algorithms Tested on UAV Images}

M. Zeybek [10] used Pix4DMapper (SFM) to create Points Cloud 3D models based on UAV images. Global

Mapper software was used to detect and measure damages. Four features (diameter, perimeter, length and depth) were measured on four potholes and seven ruts. Software measurement was compared to data collected from a survey. Software errors ( $M$ soft - $M$ field) and RMSE values are illustrated in Table VIII. M. Zeybek [10] proved also that measurement precision increases and error from field measurement decreases when the density of Points Cloud becomes large. As mentioned in Section III, the density of Points Cloud depends on the GSD value which depends on the UAV altitude. Four UAV GSD configurations were studied:

- 5mm GSD configuration presents the minor RMSE values: RMSE (diameter) $=4.41 \mathrm{~cm}$, RMSE (perimeter) $=0.03 \mathrm{~cm}$ and RMSE (depth) $=0.03 \mathrm{~cm}$;

- $10 \mathrm{~mm}$ GSD configuration: RMSE (diameter) = 8.53cm, RMSE (perimeter) $=4.18 \mathrm{~cm}$ and $\operatorname{RMSE}$ (depth) $=$ $0.03 \mathrm{~cm}$;

- $15 \mathrm{~mm}$ GSD configuration: RMSE (diameter) = $16.29 \mathrm{~cm}$, RMSE (perimeter) $=6.08 \mathrm{~cm}$ and RMSE (depth) $=0.03 \mathrm{~cm}$;

- 20mm GSD configuration presents the higher RMSE values: RMSE (diameter) $=20.38 \mathrm{~cm}$, RMSE $($ perimeter $)=6.72 \mathrm{~cm}$ and RMSE $($ depth $)=0.05 \mathrm{~cm}$.

TABLE VIII. ERRORS AND RMSE FOR DISTRESS MEASURED BY GLOBAL MAPPER SOFTWARE [10]

\begin{tabular}{|l|l|l|l|}
\hline $\begin{array}{l}\text { MEASUREMENT } \\
\text { TYPE }\end{array}$ & SAMPLE & $\begin{array}{l}\text { ERROR INTERVAL } \\
(\mathbf{c m})\end{array}$ & RMSE \\
\hline Diameter & 4 Potholes & $0.1-1.5$ & 0.010 \\
\hline Depth & $\begin{array}{l}\text { 4 Potholes } \\
\text { and } \\
7 \text { Ruts }\end{array}$ & $0.1-3.7$ & 0.009 \\
\hline Length & 7 Ruts & $2.0-15.5$ & 0.301 \\
\hline Perimeter & 4 Potholes & $13.4-32.2$ & 0.076 \\
\hline
\end{tabular}

R. Roberts [21] used DJI Mavic 2 Pro (UAV) images to measure distress. During the 3D Cloud conception, the GSD was fixed on $0.97 \mathrm{~mm} /$ Pixel to get 90508878 Points Cloud. RMSE values for Control Points and Check Points on the three3 axes (X, Y, Z) were measured. RMSE range was between $0.0078 \mathrm{~m}$ and $0.0119 \mathrm{~m}$.

\section{E. Algorithms Tested on GPR Images}

J. Gao [13] proposed the Faster R-ConvNet method, which was trained and tested on a GPR images dataset contains four damages:

- Reflection crack: $\operatorname{Pr}=88.31 \%$ and $\operatorname{Rec}=89.04 \%$;

- Water-damage pit: $\operatorname{Pr}=90.56 \%$ and $\operatorname{Rec}=89.68 \%$;

- Uneven settlement: $\operatorname{Pr}=88.51 \%$ and $\operatorname{Rec}=91.04 \%$;

- Overall: $\operatorname{Pr}=87.13 \%$ and Recall $=89.92 \%$.

M. E. Torbaghan [14] proposed a method for automatic crack detection based on GPR images. Table IX summarizes the ability of the method to detect cracks on raw and processed images. It was observed that on raw images, cracks width inferior to $8.6 \mathrm{~mm}$ cannot be detected, between $8.6 \mathrm{~mm}$ and $16.5 \mathrm{~mm}$ were hard to detect and only superior to $31 \mathrm{~mm}$ can be detected easily. However, on processed images, it was possible to detect cracks from 7mm width. On the other hand, PSNR (Peak-Signal-to-Noise Ratio) was analyzed for slabs uncovered and covered (with papers, thin layers of asphalt and filled with sand). PSNR for small-width cracks can't be analyzed.

\section{F. Transfer Learning}

To assure a good training for algorithms and reduce the need for a big amount of data, S. Ranjbar [16] trains a group of 8 pre-trained algorithms on a dataset containing 1500 images. SqueezNet achieved the higher values of Accuracy, Pr and F1score as illustrated in Table X.

TABLE IX. The Ability of the Method to Detect GPR Images [14]

\begin{tabular}{|l|l|l|l|l|}
\hline $\begin{array}{l}\text { Crack } \\
\mathbf{N}^{\circ}\end{array}$ & $\begin{array}{l}\text { LOCATION } \\
(\mathbf{c m})\end{array}$ & $\begin{array}{l}\text { WIDTH } \\
(\mathbf{m m})\end{array}$ & $\begin{array}{l}\text { RAW } \\
\text { IMAGES }\end{array}$ & $\begin{array}{l}\text { PROCESSED } \\
\text { IMAGES }\end{array}$ \\
\hline 1 & 24.0 & 14.4 & Hard & Good \\
\hline 2 & 59.0 & 1.1 & No & No \\
\hline 3 & 88.5 & 4.5 & No & Hard \\
\hline 4 & 110.0 & 16.5 & Hard & Good \\
\hline 5 & 136.5 & 10.1 & Hard & Good \\
\hline 6 & 161.0 & 8.6 & No & Hard \\
\hline 7 & 192.0 & 1.3 & No & No \\
\hline 8 & 210.0 & 31.3 & Good & Good \\
\hline 9 & 239.0 & 3.4 & No & Good \\
\hline 10 & 258.0 & 4.2 & No & No \\
\hline 11 & 265.0 & 5.7 & No & Hard \\
\hline 12 & 294.0 & 7.0 & No & Good \\
\hline 13 & 317.0 & 34.2 & Good & Good \\
\hline 14 & 371.0 & 4.7 & No & Hard \\
\hline
\end{tabular}


TABLE X. COMPARISON OF PRE-TRAined ALgORITHMS ON THE DATASET STUDY[16]

\begin{tabular}{|l|l|l|l|}
\hline $\begin{array}{l}\text { PRE-TRAINED } \\
\text { ALGORITHMS }\end{array}$ & ACCURACY & PRECISION & F1 SCORE \\
\hline AlexNet & $97.80 \%$ & $96.70 \%$ & $96.70 \%$ \\
\hline SqueezNet & $\mathbf{9 9 . 1 0 \%}$ & $\mathbf{9 8 . 6 0 \%}$ & $\mathbf{9 8 . 6 0 \%}$ \\
\hline GoogleNet & $98.90 \%$ & $98.40 \%$ & $98.40 \%$ \\
\hline ResNet-18 & $97.90 \%$ & $96.90 \%$ & $96.80 \%$ \\
\hline ResNet-50 & $97.40 \%$ & $96.10 \%$ & $96.10 \%$ \\
\hline ResNet-101 & $97.20 \%$ & $95.80 \%$ & $95.70 \%$ \\
\hline DenseNet-201 & $98.40 \%$ & $97.60 \%$ & $97.60 \%$ \\
\hline Inception-v3 & $98.50 \%$ & $97.70 \%$ & $97.70 \%$ \\
\hline
\end{tabular}

\section{LIMITATIONS AND FUTURE RESEARCH}

\section{A. Limitations}

Despite multiple researches conducted, automatic detection and maintenance of pavement distress are still reals issues in Smarts Cities. As we reviewed in previews sections, multiples sensors, software and algorithms were employed to find optimal solutions. However, results still under the perspectives of Smarts Cities' designers. Currents solutions are suffering multiple limitations on data collection, datasets construction, algorithms training and data analysis.

1) Data collection: sensors used to collect pavement data are facing the issue of getting details about small damages $(\sim 1 \mathrm{~cm})$. Environmental changes caused directly by small damages are very low. For this reason, parameters (such as temperature, vibration, etc.) are not very significant on pavement health monitoring concept.

GPR images are constructed based on electromagnetic signals reflected from pavement surfaces. Small damages reflect very weak signals that can't be easily analyzed, even if after processing operations.

Vision-based sensors (Cameras or Smartphones) are the best tools used for pavement monitoring. However, getting information about small damages depends on the camera's parameters (resolution, focal length and FOV). Despite using a high-quality camera, we should reduce the distance from the pavement, which is not always possible during traffic.

UAV makes great progress on pavement monitoring, by its ability to move around the pavement and collect images from different distances and angles. However, UAVs are still limited on embedded sensors types and quantity, weight, minimal altitude, range and the capacity of batteries.

2) Datasets: Multiple datasets present a limited number of images and distress types. Otherwise, a few datasets contain images with complex situations such as climate effects, low lighting and small objects noise. Also, the dataset should contain images with a high density of damages and degraded conditions.
3) Data analysis: most of the researches conducted were focusing on crack damages. Other types of damage (pothole, bump, ruts, etc.) are pending more specific methods and analysis. Otherwise, most of the papers are conducted on the field of the detection level. Measurement of pavement damages' dimensions (length, depth, diameter, perimeter, etc.) is still at its beginning. Also, studies and experiences were limited to small sections of the road. To the best of our knowledge, no study was conducted on monitoring a big network of roads as expected in Smarts Cities, to evaluate the ability of the system to handle a big amount of data.

\section{B. Futures Research}

To overcome the mentioned limitations and to achieve an efficient automatic pavement distress detection and measurement in Smarts Cities, we need to open the following scopes of research:

- Developing tools and methods for collection and analyzing small pavement damages $\sim 1 \mathrm{~cm}$.

- Generalization of pavement distress detection and measurement methods for other damages types than cracks.

- The conception of pavement damages datasets, exhaustive and dense, to avoid algorithms "Overfitting”.

- Development of pavement distress detection and measurement algorithms based on "Ensemble Learning” and “Transfer Learning”.

- Extraction of pavement damages from UAV videos streaming.

- Replace using multiple software and algorithms by EndTo-End algorithms.

- Development of a GPS navigation system that can alert drivers about road damages.

- Using as a low-cost solution, Backup cameras and GPS navigation systems of cars to conduct pavement surface conditions.

- Development of a "User-Friendly" smartphone application to help drivers to avoid road damages.

- Using Google Earth images to enhance pavement distress datasets quality for a large roads network.

\section{DISCUSSION}

Multiple software and algorithms were trying to extract meaningful information about pavement damage. Recently, Machine Learning and Deep Neural Networks are methods that achieved better results. The efficiency of these methods depends on the quality of data collected and algorithms training conduction. Multiple datasets were collected using fixed or mobile sensors to help with algorithms training, evaluation and testing. Several free datasets were collected under different conditions: weather, day/night, noise and data-making confusion (land markers, small objects, dust, etc.). 
Until today, there are no robust methods or algorithms that can extract all existing distress, including small damages, on a big network of roads. Such a situation is sometimes due to the poor quality of data collected, the lack of training conducted for algorithms, or the non-possibility to handle a big amount of data at a determined timing.

Our concept will focus on using multiple UAVs (Unmanned Aerial Vehicules) to monitor roads surface. Videos will be taken from different altitudes and angles by multiple sensors. UAVs will be managed to capture all data required and transmit it to a ground station for validation or rejection. A pre-trained algorithm will be used to extract pavement damages from frames' videos. Fig. 1 illustrates the flowchart of our study.

\section{CONCLUSION}

Automatic Pavement Distress Detection and Measurement is one of the most challenges in Smarts Cities. PMS (Pavement Management System) aims to keep road surfaces healthy and available all the time. For this reason, at each moment, pavement condition and futures evolution should be defined with high accuracy. We keep in mind that, Smarts Cities are looking for a low-cost solution, automatic, robust and able to handle a big amount of data.

During our review of state-of-the-art methods, it was observed that most of the papers were limited to the detection and measurement of specific damages on small pavement sections. Also, small damages were hard to analysis too and training still a real issues that needs to be overcome.

In Smarts Cities, roads networks are huge. For this reason, we need to deploy multiples sensors, to enhance the situation awareness about the health of road surfaces. The use of UAVs gives the flexibility to move around the three dimensions and change distances/angles from the pavement. Frames extracted from videos are containing meaningful information than simple images. To avoid overload, frames can be filtered before distress extraction operation.

Training of the algorithm is a critical operation that could be conducted periodically. Algorithm detection can be examined during surveys until detection achieves the accuracy and the maturity required.

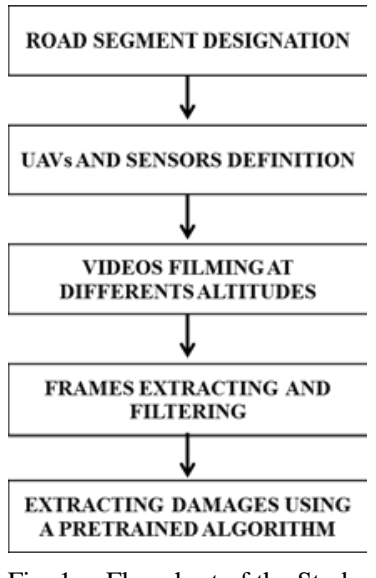

Fig. 1. Flowchart of the Study.

\section{REFERENCES}

[1] Q. Mei and M. Gu, "Multi-level feature fusion in densely connected deep-learning architecture and depth-first search for crack segmentation on images collected with smartphones”, Sage Journals, vol. 19, no 6, pp. 1726-1744, November 2020.

[2] W. Song, G. Jia, H. Zhu, D. Jia, and L. Gao, "Automated pavement crack damage detection using deep multiscale convolutional features”, Journal of Advanced Transportation, vol. 2020, Article ID 6412562, 11 pages, 2020.

[3] R. Roberts, L. Inzerillo, and G. D. Mino, "Exploiting low-cost 3D imagery for the purposes of detecting and analyzing pavement distresses”, Infrastructures Journal, vol. 5, no 1, January 2020.

[4] Z. Tong, D. Yuan, J. Gao, and Z. Wang, "Pavement defect detection with fully convolutional network and an uncertainty framework", Computer-Aided Civil and Infrastructure Engineering, pp. 1-18, 2020.

[5] Y. Du, N. Pan, Z. Xu, F. Deng, Y. Shen, and H. Kang, "Pavement distress detection and classification based on YOLO network", International Journal of Pavement Engineering, in press.

[6] Z. Fan, C. Li, Y. Chen, P. D. Mascio, X. Chen, G. Zhu, and G. Loprencipe, "Ensemble of deep convolutional neural networks for automatic pavement crack detection and measurement”, Coatings Journal, 2020.

[7] A.M. Johnson, Best practices handbook on asphalt pavement maintenance, Pub., 2000.

[8] J. Lekshmipathy, N. M. Samuel, and S. Velayudhan, "Vibration vs vision: best approach for automated pavement distress detection", International Journal of Pavement Research and Technology, vol. 13, pp. 402-410, 2020.

[9] X. Xiang, Y. Zhang, and A. El Saddik, "Pavement crack detection network based on pyramid structure and attention mechanism", IET Image Processing Journal, vol. 14, no 8, pp. 1580-1586, June 2020.

[10] M. Zeybek and S. Biçici, "Road distress measurements using UAV", Turkish Journal of Remote Sensing and GIS. vol. 1, no 1, pp. 13-23, March 2020.

[11] R. Roberts, L. Inzerillo, and G. D. Mino, "Developing a framework for using Structure-From-Motion techniques for road distress applications”, European Transport Journal, no 77, paper no 5, May 2020.

[12] Q. Mei and M. Gül, "A cost effective solution for pavement crack inspection using cameras and deep neural networks", Construction and Building Materials, vol. 256, article no 119397, September 2020.

[13] J. Gao, D. Yuan, Z. Tong, J. Yang, and D. Yua, “Autonomous pavement distress detection using ground penetrating radar and region-based deep learning”, Measurement, vol. 164, article no 108077, November 2020.

[14] M. E. Torbaghan, W. Li, N. Metje, M. Burrow, D. N. Chapman, and C. D.F. Rogers, "Automated detection of cracks in roads using ground penetrating radar", Journal of Applied Geophysics, vol. 179, article no 104118, August 2020.

[15] J. Liu, X. Yang, S. Lau, X. Wang, S. Luo, V. C. Lee, and L. Ding, "Automated pavement crack detection and segmentation based on twostep convolutional neural network", Computer-Aided Civil and Infrastructure Engineering, vol. 35, no 11, pp. 1291-1305, Novomber 2020.

[16] S. Ranjbar, F. M. Nejad, and H. Zakeri, “An image-based system for pavement crack evaluation using transfer learning and wavelet transform", International Journal of Pavement Research and Technology, vol. 14, no 4, pp. 437 - 449, July 2021.

[17] A. Ragnoli, M. Rosaria De Blasiis, and A. Di Benedetto, "Pavement distress detection methods: a review”, Infrastructures Journal, vol. 3, no 4, article no 58, December 2018.

[18] E. Ibragimov, H. J. Lee, J. J. Lee, and N. Kim, "Automated pavement distress detection using region based convolutional neural networks", International Journal of Pavement Engineering, in press.

[19] Y. Wang, K. Song, J. Liu, H. Dong, Y. Yan, and P. Jiang, "RENet: Rectangular Convolution Pyramid and Edge Enhancement Network for salient object detection of pavement cracks”, Measurement, vol. 170, article no 108698, January 2021. 
[20] S. Biçici and M. Zeybek, "An approach for the automated extraction of road surface distress from a UAV derived point cloud", Automation in Construction, vol. 122, article no 103475, February 2021.

[21] R. Roberts, L. Inzerillo, and G. D. Mino, "Using UAV based 3D modelling to provide smart monitoring of road pavement conditions", Information Journal, vol. 11, no 12, article no 568, December 2020.

[22] A. Camero and E. Alba, "Smart City and information technology: A review", Cities The International Journal Of Urban Policy And Planning, vol. 93, pp 84-94, October 2019.

[23] T. B. J. Coenen and A. Golroo, "A review on automated pavement distress detection methods”, Cogent Engineering Journal, vol. 4, no 1, 2017.

[24] X. Zhang, S. Hao, R. WenGe and C. Dave, "Intelligent transportation systems for Smart Cities: a progress review", Science China Information Sciences Journal, vol. 55, no 12, pp. 2908-2914, December 2012.
[25] Y. Guo, Z. Tang and J. Guo, "Could a Smart City ameliorate urban traffic congestion? a quasi-natural experiment based on a Smart City pilot program in China”, MDPI Sustainability Journal, vol. 12, no 6, article no 2291, March 2020.

[26] Y. Hou, Q. Li, C. Zhang, G. Lu, Z. Ye, Y. Chen, L. Wang, and D. Cao, "The state-of-the-Art review on applications of intrusive sensing, image processing techniques, and machine learning methods in pavement monitoring and analysis”, Engineering Journal, vol. 7, no 6, pp. 845856, June 2020.

[27] A. Wolters, K. Zimmerman, K. Schattler, and A. Rietgraf, Implementing Pavement Management Systems For Local Agencies, Implementation Guide, Pub. August, 2011.

[28] N. Vitillo, "Pavement Management Systems overview". 21 December, 2015.

[29] AASHT Joint Task Forces On Pavement, Guidelines on Pavement Management, Pub. 1985. 